Regular Paper

\title{
Generator Characteristics of an Interior Permanent Magnet Machine Designed by a Topology Optimization with GA Considering Cluster of Materials
}

\author{
Takeo ISHIKAWA ${ }^{* 1}$, Shota MIZUNO*1 ${ }^{* 1}$ and Nobuyuki KURITA ${ }^{* 1}$
}

\begin{abstract}
This paper designs the rotor structure of a permanent magnet (PM) synchronous generator by a topology optimization method. The implemented optimization method is the Genetic algorithm coupling the concept of cluster of materials and a cleaning procedure, and then it assumes four hexahedron PMs similar to the rotor shapes obtained by the proposed method for reasons of manufacturing ease. This paper manufactures the optimized rotor structure, which has a high flux linkage. Experimental results clarify that the voltage regulation is improved and that the efficiency is improved.
\end{abstract}

Keywords: Interior permanent magnet generator, topology optimization, Genetic algorithm, voltage regulation, efficiency.

(Received: 24 July 2014, Revised: 7 April 2015)

\section{Introduction}

Permanent magnet (PM) synchronous machines are widely used as motor/generator in hybrid vehicles and electric vehicles and so on, because of their high efficiency and high power density. A large variety of PM rotor topology have been developed for high output power, for example, surface permanent magnets (SPMs) and several types of interior permanent magnets (IPMs), because the rotor geometry of the PM machine greatly affects the machine performance. In order to get a good PM machine, it is very important to develop an initial conceptual structural topology of the PM rotor. For this purpose, several kinds of topology optimization procedure have been proposed, because they are promising. Topology optimization allows one to obtain an initial conceptual structure starting with minimal information on the object's structure.

The authors proposed a topology optimization method to optimize the distribution of materials within an electrical machine using the Genetic algorithm (GA) [1]. The concept of a cluster and cleaning procedure of material was introduced to the method proposed in [1] and a stator of brushless DC motor was designed [2]. It considered only two kinds of material, air and iron, and is therefore similar to the ON/OFF method [3-5]. And then, the previous method was improved so as to take into account more than two kinds of material, namely, air, iron, $r$-oriented, $x$-oriented and $y$-oriented magnets [6]. Moreover, the obtained rotor structure was assumed to consist of simple shape of PMs in order to consider ease of manufacturing [7].

\footnotetext{
Correspondence: T. ISHIKAWA, Division of Electronics and Informatics, Gunma University. 1-5-1 Tenjin-cho, Kiryu, Gunma 376-8515, Japan

email: ishi@el.gunma-u.ac.jp

${ }^{* 1}$ Gunma University
}

Chromosome Gene locus
\begin{tabular}{|c|c|c|c|c|c|c|c|c|}
\hline parent 1 & 0 & $\mathbf{0}$ & 1 & 1 & $\mathbf{2}$ & 2 & $\mathbf{\ldots}$ & $\mathbf{3}$ \\
\hline parent 2 & 2 & $\mathbf{2}$ & 3 & 3 & $\mathbf{1}$ & 1 & $\mathbf{.}$ & $\mathbf{0}$ \\
\hline & $\uparrow$ & \multicolumn{7}{c|}{$\uparrow$} \\
\hline child 1 & 0 & $\mathbf{2}$ & 1 & 1 & $\mathbf{1}$ & 2 & $\ldots$ & $\mathbf{0}$ \\
\hline child 2 & 2 & $\mathbf{0}$ & 3 & 3 & $\mathbf{2}$ & 1 & $\cdots$ & $\mathbf{3}$ \\
\hline
\end{tabular}

Fig.1. An example of genes and the uniform crossover in the GA.

This paper applies the proposed topology optimization method to design of a rotor structure of PM synchronous generator. The optimized rotor structure is manufactured, and the performance of the manufactured IPM synchronous generator is clarified.

\section{Design by the Proposed Topology Optimization Method}

Here we briefly explain the topology optimization method which was proposed in [6] and is to be implemented in this paper. GA is a method which imitates the evolution of living things, and is suitable for problems containing a large search extent. In the proposed method, a design region is discretized into finite element meshes and the material of several elements, say, a cell, is set to a gene in chromosome. If we consider three kinds of material, for example, air, iron and magnet, which are set to 0,1 and 2 , respectively, the chromosome is composed of some genes as shown in Fig.1. Two parents are randomly selected and some genes are selected to be exchanged by a uniform crossover with a crossover ratio, and then two new children are generated as shown in Fig. 1. Children inherit good fitness of parents by repeating the process. Fig. 2 shows a cross 


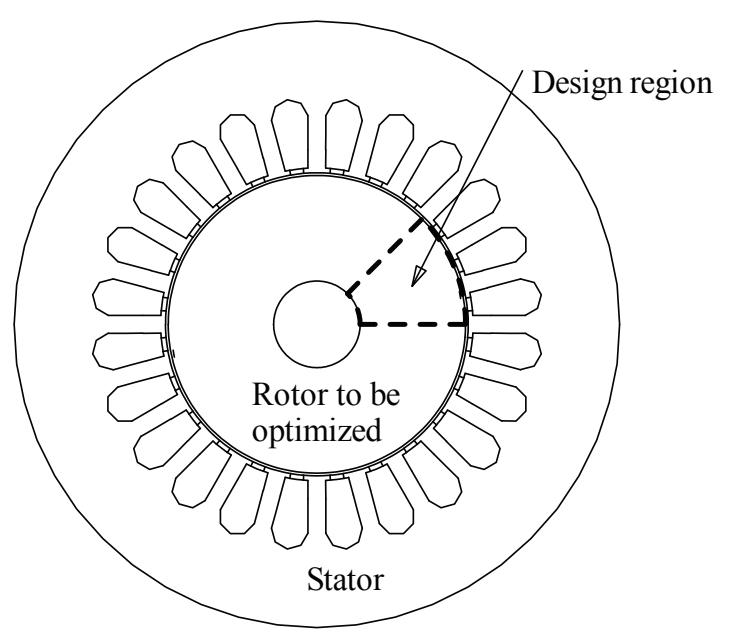

Fig. 2. Cross section and design region of a generator.

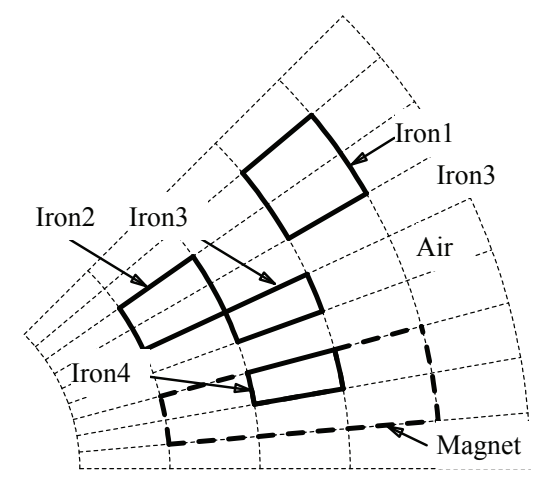

(a) Before cleaning procedure

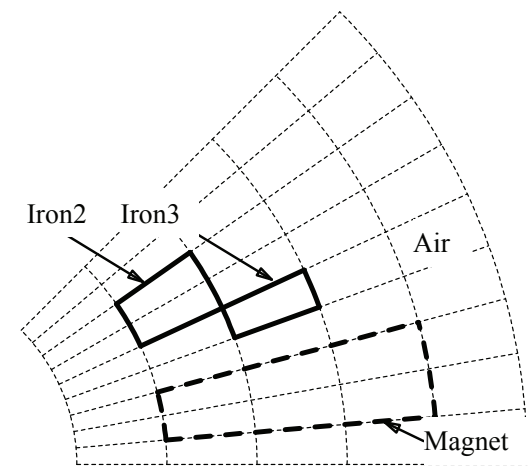

(b) After cleaning procedure

Fig. 3. A cluster of materials and the cleaning method.

section of four-pole PM synchronous generator with distributed windings. One eighth of the rotor is designed because of symmetry, and is divided to 5 by 9 array of cells as shown in Fig. 3. We proposed a concept of cluster of many kinds of material. Here, the cluster means a group of cells which have same material. For example, irons 2 and 3 form the same cluster, and iron 1 forms another cluster in Fig. 3(a). If the size of cluster is small, that is, the number of cells in the region is smaller than or equal to an integer $N_{\min }$, a cleaning procedure is carried out. Then, irons 2 and 3 remain, and other irons are dismissed. Iron 1 is changed to the surrounding material, air, and iron 4 is changed to magnet as shown in Fig. 3(b). This cleaning procedure for the small cluster of material can remove the floating pieces of material. This paper iterates the GA with a newly increased length of genes. At the 1st iteration, a rough topology is designed using a small number of design variables, that is, 5 by 9 array of cells. Then, a fine topology is designed using a large number of design variables for the next iteration, that is, 20 by 18 array of cells. For the 2nd and 3rd iterations, a set of initial individuals in the GA inherit the individual which has the best fitness at the conclusion of previous iteration.

In our past research, the rotor structure of PM synchronous motor was designed. The fitness function in the GA was defined as follows to acquire the structure for large average torque and to restrain the volume of PM,

$$
\text { fitness }=\frac{T_{\text {ave }}}{k V_{p m} / V_{\text {rotor }}+1}
$$

where, $T_{\text {ave }}, V_{p m}, V_{\text {rotor }}$ and $k$ are the average torque, the volume of PM, the volume of rotor, and a constant, respectively. The fitness function becomes small if $V_{p m}$ is large, because $V_{\text {rotor }}$ is constant. If $k$ is large, the weighting of the PM volume to the fitness function becomes large. The torque of IPM synchronous motor is expressed by

$$
T_{\text {ave }}=p \Phi I \cos \beta+0.5 p\left(L_{q}-L_{d}\right) I^{2} \sin 2 \beta
$$

where $p, \Phi, I$ and $\beta$ are the number of pole pairs, flux linkage, stator current and phase angle of stator current, respectively. If the stator current $I$ is constant and the phase angle $\beta$ is $0, T_{\text {ave }}$ is proportional to the flux linkage $\Phi$, resulting in the electromotive force $E$, when the motor speed is constant. Therefore, equation (1) is the same as

$$
\text { fitness }=\frac{E}{k V_{p m} / V_{\text {rotor }}+1}
$$

This fitness function can give a structure for higher electromotive force under restraining the volume of PM.

This paper optimizes the topology of the rotor structure of a PM synchronous generator by considering two cases. The first case considers three kinds of material, namely, air, iron and $r$-oriented magnet, and the second one considers four kinds of material, namely, air, iron, and both $x$ - and $y$-oriented magnets. In the optimization process, $k$ is set to be 5 , and the number of populations is set to be 45 . If the number of populations is larger than 45 , there is a possibility to get a better result. However, this would lead to a longer computational time.

Fig. 4 shows an obtained rotor structure, where three kinds of material, namely, air, iron and the $r$ oriented magnet are taken into account. $N_{\min }$ is set to be 1 for air and iron at the 1st iteration. This means that if the number of cells is equal to 1 , the material of the cell is changed to the surrounding material. $N_{\min }$ is set to 4 at the next iteration. It is found that the consideration of $r$ oriented magnet gives a kind of surface permanent 


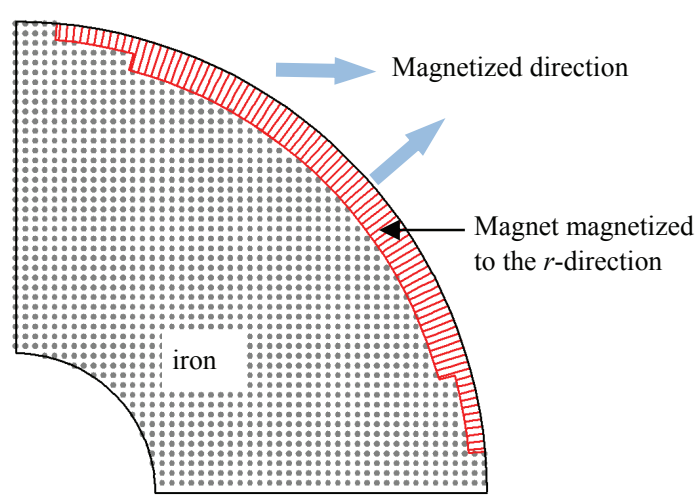

Fig. 4. Rotor structure obtained by considering air, iron and $r$-oriented magnet.

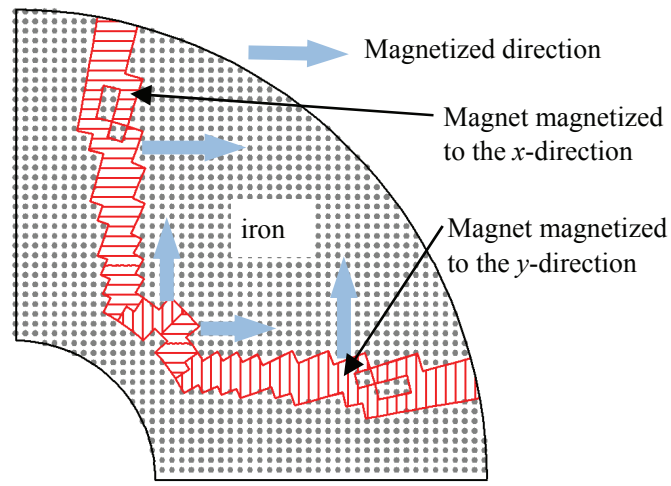

Fig. 5. Rotor structure obtained by considering air, iron and $x$ - and $y$-oriented magnets.

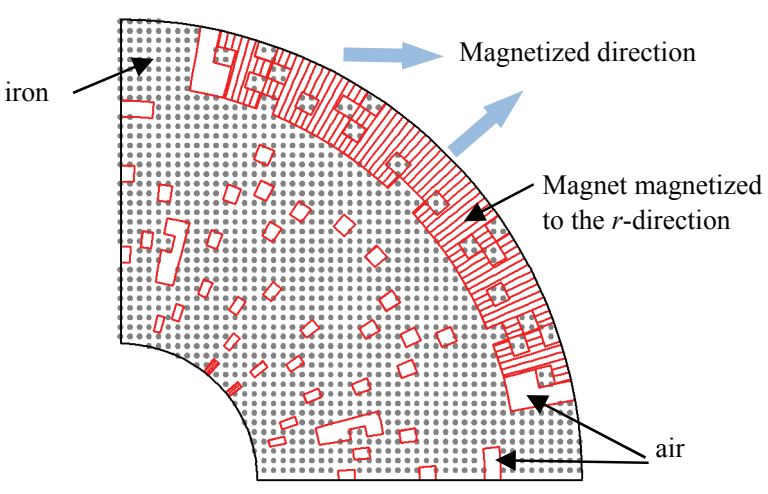

Fig. 6. Rotor structure obtained by considering air, iron and $r$-oriented magnet without considering the cleaning procedure.

magnet type rotor. Fig. 5 shows a rotor structure obtained by considering $x$-and $y$-oriented magnets. The rotor shape is a kind of interior permanent magnet type rotor, whose shape looks like "V" or "W". Fig. 6 shows an obtained rotor structure, when the cluster of material and the cleaning method is not carried out. Although the obtained magnet shape looks similar to the one shown in Fig. 4, the rotor has lots of small pieces of iron in the $r$ oriented magnet. Therefore, this rotor has a very

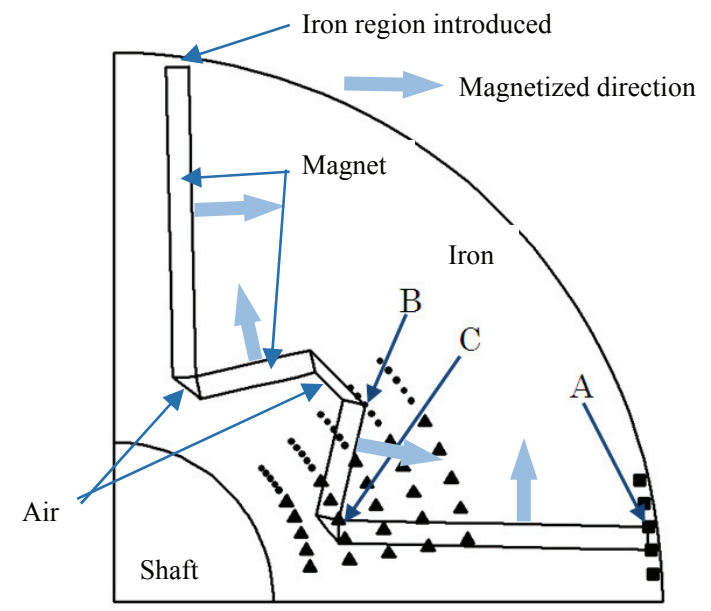

Fig. 7. Rotor structure represented by a simple magnet shape.

complicated and weak structure, and is difficult to manufacture.

\section{Optimization Taking into Account Ease of Manu- facturing}

The rotor structures obtained in the previous chapter are complex and impractical. In order to take into account ease of manufacturing, magnets and air-regions are assumed as simple shapes and then are optimized by using conventional optimization techniques. Since the fitness function for the rotor shown in Fig. 5 is larger than that in Fig. 4, this paper optimizes the rotor structure shown in Fig. 5. The rotor structure shown in Fig. 5 can be assumed to contain four hexahedron PMs shown in Fig. 7. In this shape, the rotor structure is represented by six parameters $\left(r_{A}, \theta_{A}, r_{B}, \theta_{B}, r_{G}, \theta_{C}\right)$ if the thickness of magnets are the same as each other and the volume of PMs is specified. This paper assumes that each magnet is magnetized in the vertical direction as shown in Fig. 7 for ease of magnetization. Moreover, this paper assumes that core area is introduced at the surface of the rotor so that the machine strength is insured against centrifugal force. Therefore, the radius of point $\mathrm{A} r_{A}$ is fixed, and then the number of design parameters is five.

This paper designs the rotor of an initial IPM machine. This initial machine was manufactured by a company, and is well known as a compressor motor for an air conditioner in Japan. The ratings of the initial motor are $1.5 \mathrm{~kW}, 3,000 \mathrm{~min}^{-1}, 192 \mathrm{~V}, 5.6 \mathrm{~A}$, and the main dimensions are stator diameter of $112 \mathrm{~mm}$, rotor diameter of $55 \mathrm{~mm}$ and air-gap length of $0.5 \mathrm{~mm}$. This paper uses a full search method to optimize the rotor shape, because we would like to verify that there is a good rotor shape similar to that shown in Fig. 5. The parameters $\theta_{A}, r_{B}, \theta_{B}, r_{C}, \theta_{C}$ have five candidates respectively as shown by points in Fig. 7, namely, $5^{5}=$ 3,125 patterns are calculated in the full search method. 


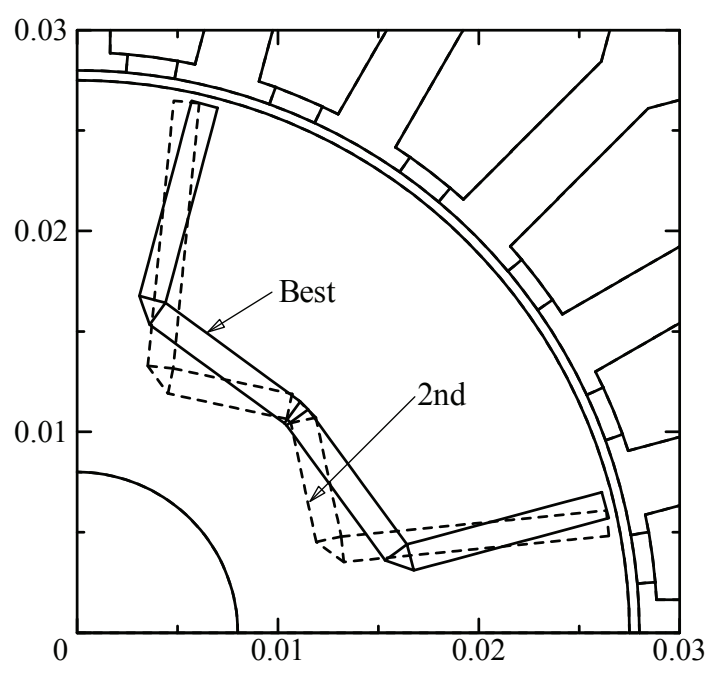

Fig. 8. Obtained rotor structure.

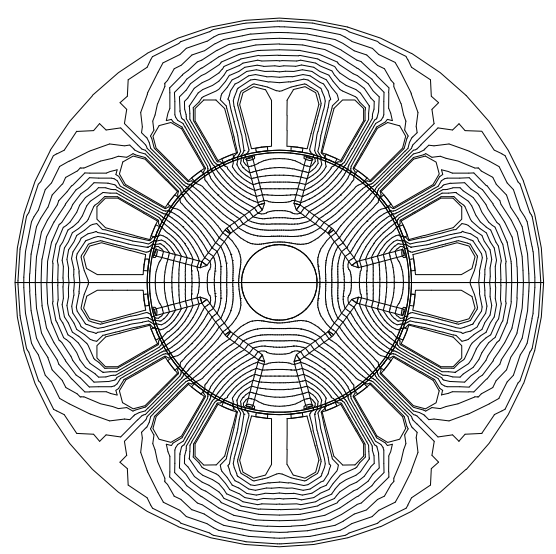

(a) Designed generator

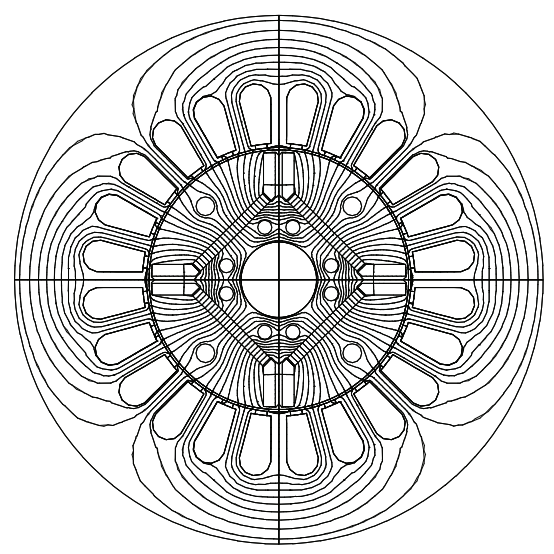

(b) Initial generator

Fig. 9. Obtained rotor structure and flux distribution at no-load.

Moreover, this paper iterates the full search method twice, where the search area of the parameters was set to about half from the first iteration. The finite element mesh is automatically

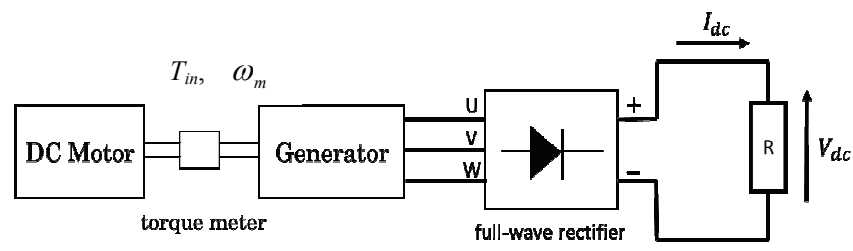

Fig. 10. Experimental set up for generator.

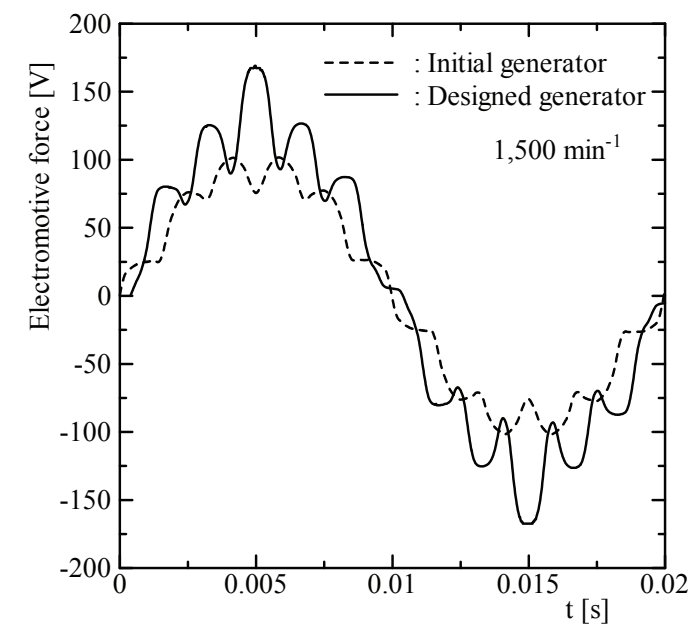

Fig. 11. Measured electromotive force at no-load.

generated by the Delaunay method in the full search method.

Fig. 8 shows the obtained rotor structures. Here, the "best" and "2nd" rotor structures are shown. It is found that the best shape is " $U$ " type and the second best shape is "W" type. Fig. 9(a) shows the flux distribution at noload. Fig. 9(b) shows the flux distribution of an initial machine whose stator is the same as Fig. 9(a). It is found that the flux is increased by about $40 \%$ because of the long and thin magnets of the optimized rotor. The calculated flux linkage of "2nd" rotor structure is almost the same as that of "best" structure. This means that the shape of PM in the rotor inside is not very important for the flux linkage.

\section{Performance of the Designed IPM generator}

We have manufactured the designed rotor shown in Fig. 9(a). Fig. 10 shows an experimental setup to measure the performance of generators. The generator is rotated by a DC motor, and the output is rectified by a full bridge rectifier and is connected to a resister. Fig. 11 shows the measured electromotive forces of the designed generator and the initial generator at a rotating speed of 1,500 $\mathrm{min}^{-1}$. They include a harmonic component, and the value of the electromotive force of the designed generator is higher than that of initial one. Table 1 shows the effective value and some components of electromotive force. It is found that the electromotive force is 1.4 times as high as that of the initial one. However, it includes a high 11-th harmonic component. 
Table 1 Measured electromotive force at no-load.

\begin{tabular}{|c|c|c|c|}
\hline & $\begin{array}{c}\text { Initial } \\
\text { generator }\end{array}$ & $\begin{array}{c}\text { Designed } \\
\text { generator }\end{array}$ & Ratio \\
\hline Effective value [V] & 68.2 & 96.3 & 1.41 \\
\hline $\begin{array}{c}\text { Fundamental } \\
\text { component [V] }\end{array}$ & 95.6 & 133.8 & 1.40 \\
\hline $\begin{array}{c}\text { 11-th harmonic } \\
\text { component [V] }\end{array}$ & 11.3 & 21.0 & 1.85 \\
\hline
\end{tabular}

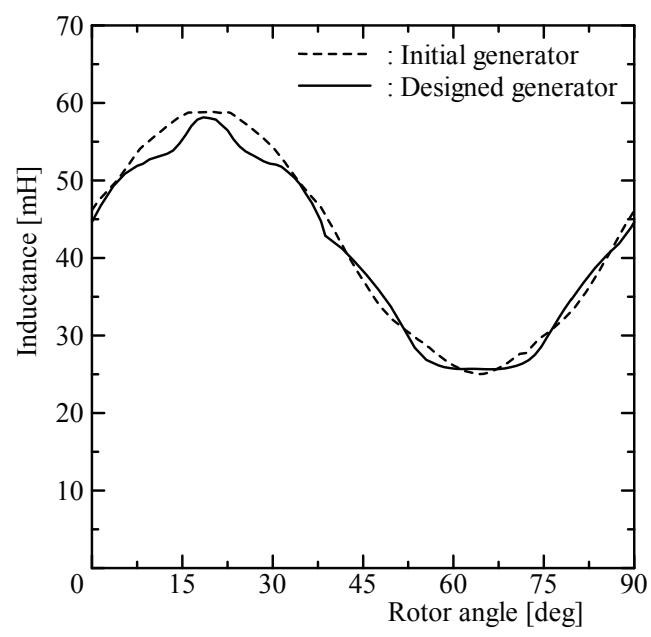

Fig. 12. Inductance characteristics.

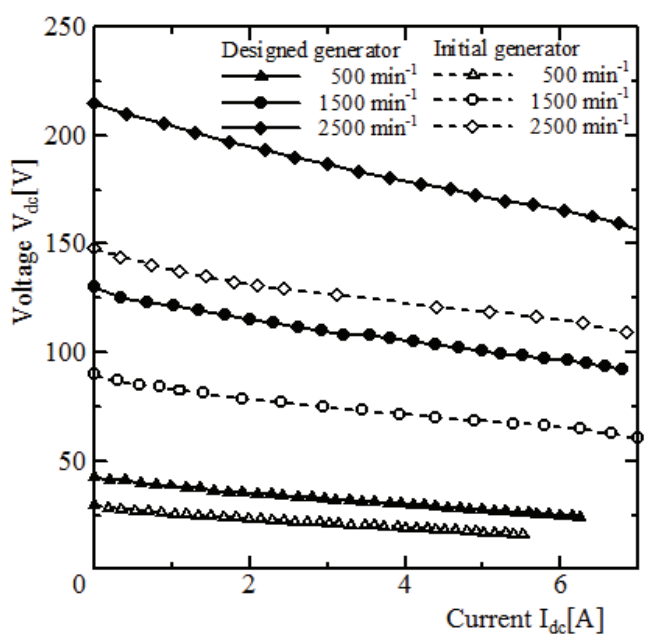

Fig. 13. Load characteristic.

Fig. 12 shows inductances measured by an LCR meter at $100 \mathrm{~Hz}$. From this figure, $d$ and $q$ inductances $L_{d}$ and $L_{q}$ are 12.7 and $28.4 \mathrm{mH}$ for the designed generator, and 12.5 and $29.4 \mathrm{mH}$ for the initial one, respectively. The saliency ratio $L_{q} / L_{d}$ of 2.24 of the designed generator is a little larger than that of the initial one of 2.35, but these two are approximately same as each other.

Next, in order to clarify the voltage regulation, this paper has measured the load characteristic by changing the load resistance, and shows the result in Fig. 13. $V_{d c}$ of the designed generator is higher than that of the initial one, because $V_{d c}$ corresponds to the rectified electromotive forces when the current $I_{d c}=0$. The DC
Table 2 Voltage regulation.

\begin{tabular}{|c|c|c|}
\hline Speed $\left[\mathrm{min}^{-1}\right]$ & $\begin{array}{c}\text { Initial generator } \\
{[\%]}\end{array}$ & $\begin{array}{c}\text { Designed genera- } \\
\text { tor [\%] }\end{array}$ \\
\hline 500 & 82.8 & 62.7 \\
\hline 1500 & 35.6 & 31.9 \\
\hline 2500 & 27.1 & 27.3 \\
\hline
\end{tabular}

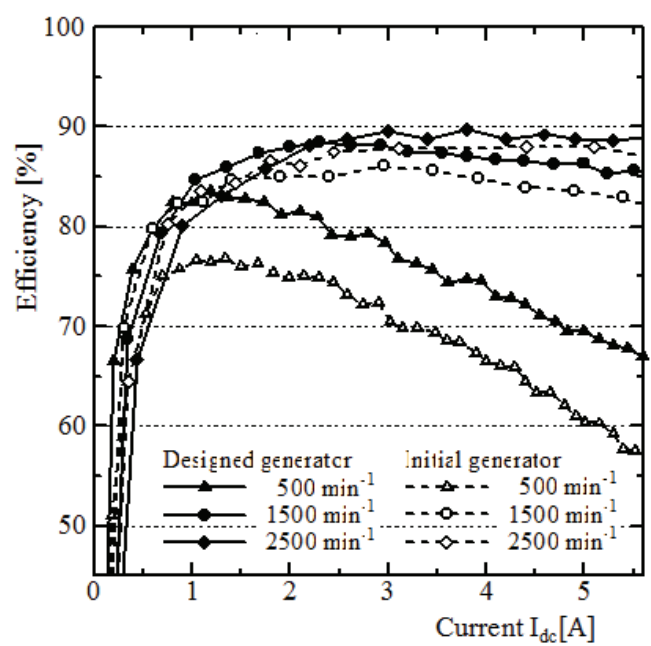

Fig. 14. Efficiency.

voltage $V_{d c}$ is reduced when the DC current $I_{d c}$ becomes large. The voltage regulation is shown in Table 2. Here, this paper assumes that the rated condition is a situation when $I_{d c}$ is equal to the rated stator current. Although the voltage regulation of the designed generator is approximately the same as that of the initial one in a high speed range, it is smaller, that is, better than the initial one when the speed is not so high.

Fig.14 shows the measured efficiency. Here, the efficiency is defied by

$$
\eta=\frac{V_{d c} I_{d c}}{T_{i n} \omega_{m}}
$$

where $T_{i n}$ and $\omega_{m}$ are the torque measured by a torque meter and the generator speed, respectively. It is found that the efficiency is high in a wide output current range and in a wide speed range. The efficiency can be expressed by the ratio of the output power to the output power plus loss. And if we ignore the iron loss $W_{\text {iron }}$, the efficiency becomes

$$
\eta=\frac{V_{d c} I_{d c}}{V_{d c} I_{d c}+2 R_{1} I_{d c}^{2}+W_{\text {iron }}}=\frac{1}{1+2 R_{1} I_{d c} / V_{d c}}
$$

where $R_{1}$ is the stator resistance. As $V_{d c}$ of the designed generator is higher than that of the initial one, the efficiency of the designed generator becomes higher. The maximum efficiency of the designed generator is about $2 \%$ higher than that of the initial one.

\section{Conclusion}

This paper has designed the rotor structure of PM synchronous generator. The implemented topology optimization is the GA using the concept of cluster of 
materials and the cleaning procedure. And then this paper has assumed four hexahedron PMs similar to the rotor shapes obtained by the proposed method for reasons of manufacturing ease. We have manufactured the designed rotor, which have a high flux linkage. It has been clarified that the voltage regulation is improved, and that the efficiency is improved by about $2 \%$.

\section{Acknowledgment}

This work was supported by JSPS KAKENHI Grant Number 25420241.

\section{References}

[1] T. Ishikawa, M. Kaneda, and A. Yamagiwa, "Optimal material distribution design of interior permanent magnet synchronous motor using Genetic algorithm," The 10th biennial IEEE CEFC, No.P2-4, 2002.

[2] T. Ishikawa, K. Yonetake, and N. Kurita, "An optimal material distribution design of brushless DC motor by Genetic algorithm considering a cluster of material," IEEE Trans. on Magn., Vol.47, No.5, pp.1310-1313, 2011.

[3] C. H. Im, H. K. Jun, and Y. J. Kim, "Hybrid genetic algorithm for electromagnetic topology optimization," IEEE Trans. on Magn., Vol.39, No.5, pp.2163-2169, 2003.

[4] J. S. Choi and J. Yoo, "Structural topology optimization of magnetic actuators using Genetic algorithms and ON/OFF sensitivity," IEEE Trans. on Magn., Vol.45, No.5, pp.2276-2279, 2009.

[5] N. Takahashi, T. Yamada, and D. Miyagi, "Examination of optimal design of IPM motor using ON/OFF method," IEEE Trans. on Magn., Vol.46, No.8, pp.3149-3152, 2010.

[6] T. Ishikawa and K. Nakayama, "Topology Optimization of Rotor Structure in Brushless DC Motor with Concentrated Windings Using Genetic Algorithm Combined with Cluster of Material," IEEE Trans. on Magn., Vol.48, No.2, pp.899-902, 2012.

[7] T. Ishikawa, K. Nakayama and N. Kurita, "Optimization of Rotor Topology in PM Synchronous Motors by Genetic Algorithm Considering Cluster of Materials and Cleaning Procedure," IEEE Trans. on Magn. Vol.50, No.2, article \# 7015704, 2014. 